\title{
Resistive Switching Behavior seen from the Energy Point of View
}

\author{
Jorge Gomez, Angel Abusleme \\ Dept. of Electrical Engineering \\ Pontificia Universidad Cat6lica de Chile \\ Santiago, Chile \\ \{jtgomezl, angel\}@uc.cl
}

\author{
Ioannis Vourkas \\ Dept. of Electronic Engineering \\ Universidad Tecnica Federico Santa Maria \\ Valparaiso, Chile \\ ioannis.vourkas@usm.cl
}

\author{
Antonio Rubio \\ Dept. of Electronic Engineering \\ Universidad Politecnica de Catalufia \\ Barcelona, Spain \\ antonio.rubio@upc.edu
}

\begin{abstract}
The technologyof Resistive Switching (RS) devices (memristors) is continuously maturing on its way towards viable commercial establishment. So far, the change of resistance has been identified as a function of the applied pulse characteristics, such as amplitude and duration. However, parameter variability holds back any universal approach based on these two magnitudes, making also difficult even the qualitative comparison between different RS material compounds. On the contrary, there is a relevant magnitude which is much less affected by device variability; the energy. In this direction, we doubt anyone so far has ever wondered "what is the quantitative effect of the iryected energy on the device state?" Interestingly, a first step was made recently towards the definition of performance parameters for this emerging device technology, using as fundamental parameter the energy. In this work, we further elaborate on such ideas, proving experimentally that the "resistance change per energy unit" ( $d R J d E)$ can be considered a significant magnitude in analog operation of bipolar memristors, being a key performance parameter worth of timely disclosure.
\end{abstract}

Keywords-memristor; resistive switching; ReRAM; device characterization; transimpedance amplifier; voltage ramp speed

\section{INTRODUCTION}

Unprecedented attention on the new and emerging technology of resistive switching (RS) devices (memristors and their relevant applications has been drawn ever since 2008 and the first demonstration of the well-known TiO2-based memristor by Hewlett-Packard Laboratories (HP Labs) [1]. Such publication managed to connect the nature of such devices with Chua's theory from 1971 about the existence of the fourth fundamental circuit element [2]. Today, memristor device technology and potential applications, including memory [3], logic [4] and unconventional computing [5], constitute an emerging trend in modem electronics.

The HP Labs' invention also concerned the development of a simple device model, which has been ever since the basis for several more behavioral and SPICE-compatible memristor models published later [6-8]; some more complex but accurate physics-based models [9] have been published as well, that go deeper into the device dynamics, hence revealing the important steps made for the continuous advancement of this technology. In this context, as far as device characterization is concerned, so far the change of resistance has been identified as a function of the applied pulse characteristics, such as amplitude and duration. However, parameter variability (e.g. for the voltage threshold values of SET and RESET processes) holds back any universal modeling approach based on these two magnitudes. What's more, it seems that variability is inevitably present in all such devices, making difficult even the qualitative comparison/selection between different material compounds that exhibit RS behavior. On the contrary, recent studies [10] have shown that there is a relevant magnitude which is much less affected by device variability (it hardly changes from cycle-to-cycle); the energy

Interestingly, a first step was recently made towards the definition of key performance parameters for this new and emerging electronic device technology, using as fundamental parameter the injected energy [11). In this direction, we doubt anyone so far has ever wondered "how much energy a RS event costs?" or even "what is the quantitative effect of the iryected energy on the device state?' This work builds upon and further elaborates on such ideas, proving experimentally that the "resistance change per energy unir' $(d R / d E)$ can be considered a significant magnitude in analog operation of bipolar memristors, being also a key performance parameter worth of timely disclosure. Measurements were made on bipolar memristor devices provided by Knowm Inc. [12] using a low cost data acquisition setup [13]. Likewise in [11], the results presented here confirmed that the injected energy is a magnitude that deserves our attention, informative enough, to be used in modeling approaches and quantitative comparisons of different memristive device families.

\section{EXPERTh1ENTAL SETUP}

Aiming to affordably get around highly expensive instrumentation requirements for memristor characterization, a low cost data acquisition setup was recently presented in [13] using a transimpedance amplifier in the target topology (see 


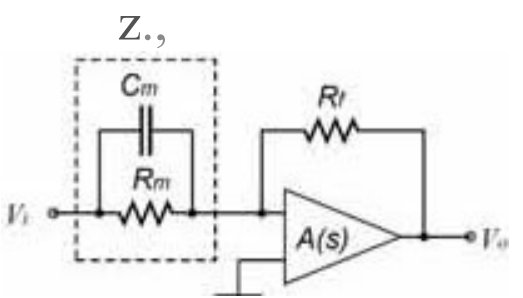

(a)

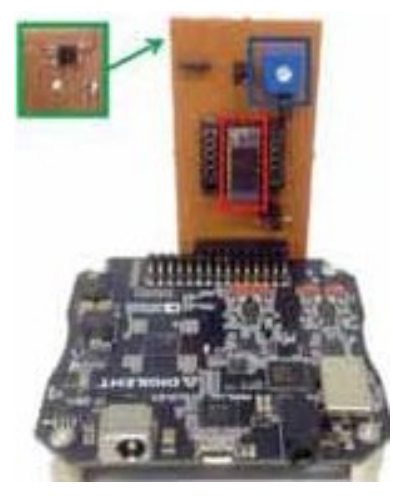

(b)

Fig. I (•) Feedback Anurntertop,lcgy[13]. The uivale1d impe,1.,...e of \&e nw.n-WStor is replt!serd:ed by $z_{\text {,y,. }}$ (b) Th.e bw ost expelimmtal setup. TI-e ref squaresh:,ws tl-e mmuistor $K n\left(. M f^{\prime}(J D I P 16\right.$ pacl:age, wlvneas; the green squ.are sho.vs tl-e operational lifier(Lxatoo. at the back side of the FCB that is connected to the Digilero AD2 tool) a:ni tl't? feedback i:esistm:e is in theb he square(J»terdiom,t«).

Fig. 1( ). Owing to feedback, this circuit generates a .,;rtual grotmd so that the memristor tmder test sees only the input voltage source and ground at its terminals. Therefore, for a given feedback resistor $R$, the current can be easily computed by measuring the output voltage $V$.. Additionally, this topology allows limitingthe current up to $/ \mathrm{m} ; \mathrm{x}$ ( currentlimit of the amplifier), which can be adjusted by chan ng the supply voltage $V s, p$ of the operational amplifier (opamp). Figure 1(b) shows the actual experimental setup of this work, based on the topology of Fig. 1(a). The BS-AF-W memristors developed by Knowm Inc are in a 16-pin DIP package. The PCB includes a MIC 7122YMM opamp for its wide $V$ sup range support, and a feedback resistor $R$,. It was connecred directly to the Digilent AD2 tool, a modem, low-cost multi-functioninstrumentation and data acquisition sysrem. All in all, such feedback allows exploring memristive behavior while having a direct control over the applied voltage and also limiting the curren guaranteeing safe experiments.

\section{Ill . EXPERIMENTALRESUL'JS}

Next we focus on measurements with pulsed SET/RESET inputs applied to Kno!Mn Inc . memristors, aiming to analyze the impact of the pulse propetties(amplirude, duration) on the device behavior. Figure 2 reveals such dependencies $O C$ conductivity change on time and voltage during the SET process. A precise RESET (proposed in [14D took place first before each experimenl Then, a 5-ms wide SET pulse of a specific amplitude was applied and the time-evolution of the resistance was monitored. The same experiment was repeated

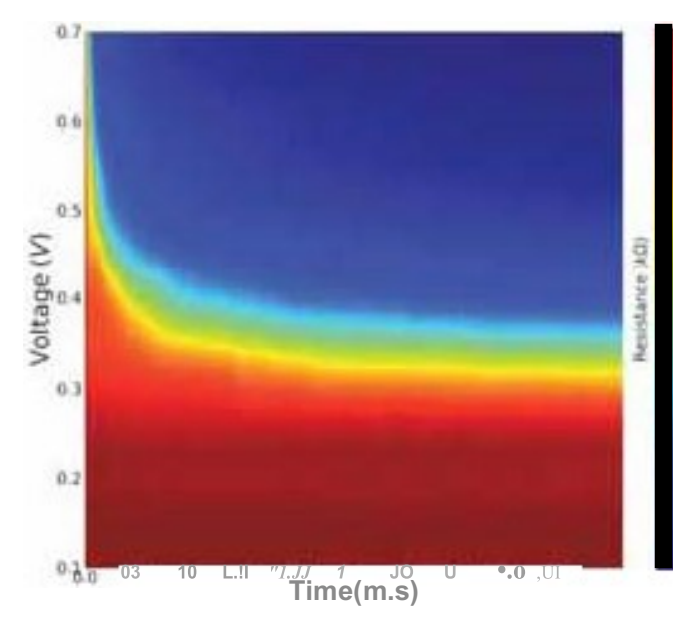

Fig. 2 ResistattO? evolution depenienoe on th? art'Plitude ani thewidthof th? applied SET Vol p.1lse. 1le device was first iro.tializoo. with precision a10.1ni 8. . Pulses of a specific artillitude ra:a .ngbetween0.1Va:aid0.7V (illci:emrnti.ngby $0.04 \mathrm{~V}$ ) wen applioo.. For eacha:anplitude, the experin'etd was eal>Id IOxamhne fu? n-eanvahesan shonn(interpolawd).

for several different amplib.lde values. So, in Fig. 2 the memristance evolution is read horizontally from left to right for a specific amplitude. It can be noticed that there is a distinguishable separation (approximately around $0.3 \mathrm{~V}$ ) between amplitudes that hardly affect the device state and those that do affect it. We call this an "exploratory performance $m a\left\{f^{\prime}\right.$ as it pennits selecting the amplitude and the width of the applied pulses depending on the nature of the target application Apparently, pulses with amplib.ldes higher than $0.5 \mathrm{~V}$ would serve only for binary switching. regardless (almost) of the selected pulse-width. On the other hand, amplitudes between $0.3 \mathrm{~V}$ and $0.5 \mathrm{~V}$ could permit multi-level tuning. whereas pulses of amplitude lower than $0.2 \mathrm{~V}$ can be used to sense the device state. Qualitatively similar results can be obtained for the RESET process, although the latter is generally considered a more complicated process. Interestingly, it seems there is not any hard SET threshold, as the borderline between amplitudes that affect and not affect the device is moving upwards for higher applied amplirudes.

Essentially, the applied square pulses have finire rise and fall times. So, increasing the amplitude of the applied square pulse while keeping its duration fixed, is equivalent to changing the gradient of the rising and falling edges; in other words, the voltage-ramp-speed (VRS) [1OJ. In this contex Fig 3 shows precisely this effect. In fac a setof240 total (120 positive and 120 negative) voltage ramps were applied, ran ng from 100 to $1200 \mathrm{~V} / \mathrm{s}$, increasing each time by I00V Is. Prior to the realization of every experimen $\backslash$ the device was SET or RESET always in the same way, to eliminate potential dependence on the initial conditions. The voltage and current were monitored throughout each experiment. The exact voltage and energy when the device completed each time the SET or RESET process, were calculated and stored. Data in Fig. 3 corresponds to the mean value of 10 experiments for each VRS value.

It can be observed that, the higher the VRS, the higher the absolure voltage value necessary to complete the SET or RESET process. In this conrext, we assumed that SET/RESET 


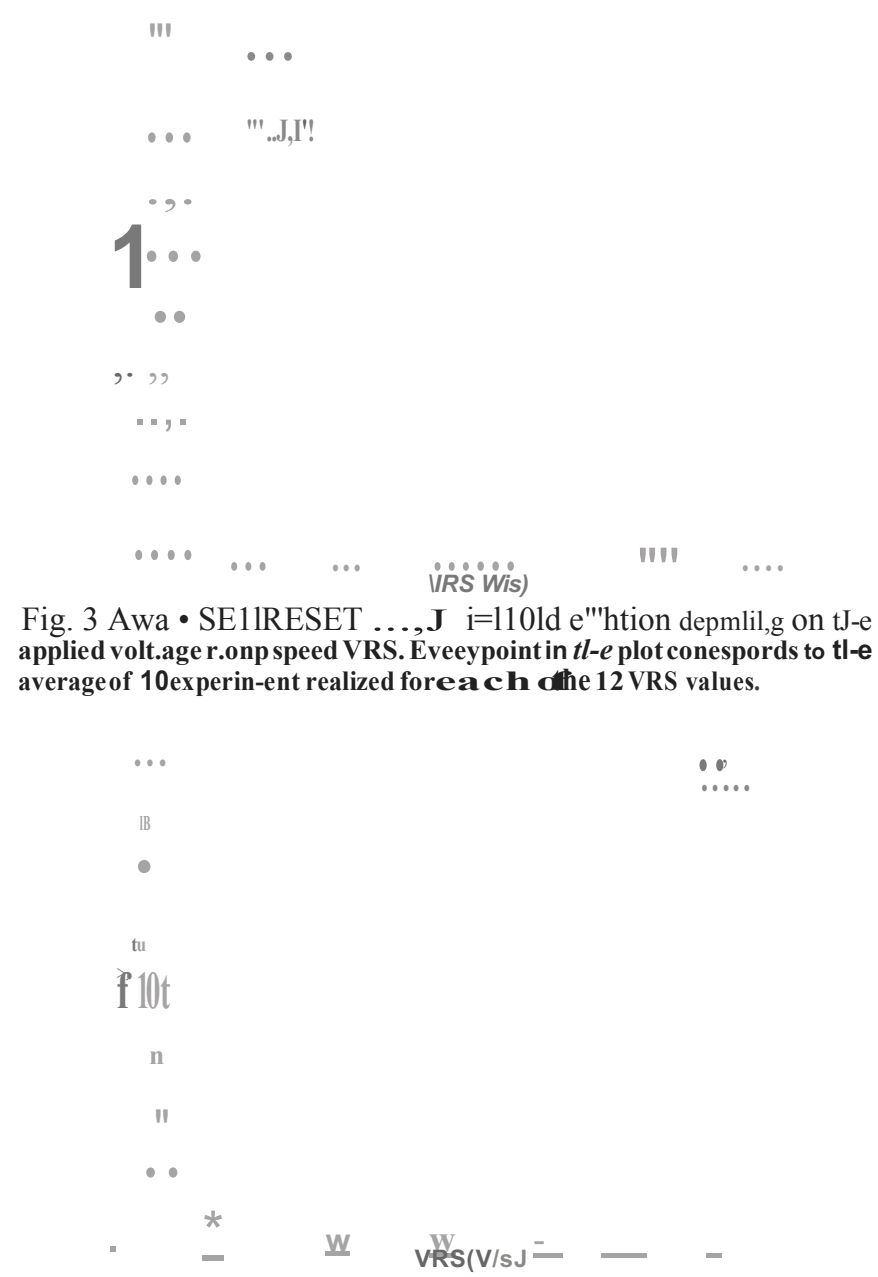

Fig. 4 Awa SE1YRESET en?igy i:equired to trigger tl-e RS eveltt, depenling on tl-e applied volt.age speed VRS. Eveeyp:,Ult in tl-.? pbt conesponds to the a.vei:age of 10 experimrnts iealizoo foreachone of the 12 VRS Vams.

was successful when the resistance was below $2 \mathrm{kQ} /$ above 3 $\mathrm{kQ}$, respectively, leaving in-between a guard band(undefined state) of I kQ. Such threshold selection was simply based on the inherent device behavior, since the switching process always involves a "resistance jump" in this resistance range, making it easier to identify SET/RESET events during data processing Such findings explain why selecting amplitude w.r.t. time (or vice versa) separately, might not be really a comprehensive strategy, being also susceptible to device variability.

The question now is: "is there any more comprehensive magnitocle Iv take into consideration IIWlile doing this exploration?' In such quest., we show in Fig. 4 that there is indeed such a magnitude that is much less affected by device variability and also unifies/simplifies such exploratoiy analysis, combining the effect of voltage and time in a single unit; it is the il)jected enetyyrequired to trigger the RS evenl More specifically, Fig. 4 shows in fact that the averago energy injected during a SET process is nearly constan independent of the frequency of the applied pulsed sii,iials. This is more notable for the SET process, whereas for the RESET it applies onlyfor high VRS values; i.e., when the applied square pulse is closer to an "ideal" pulse with veiy short rise/fall times.

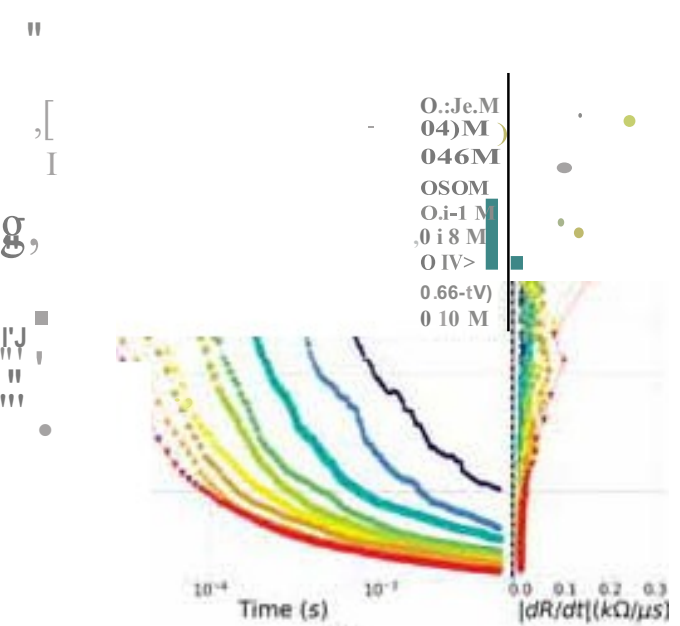

(a)

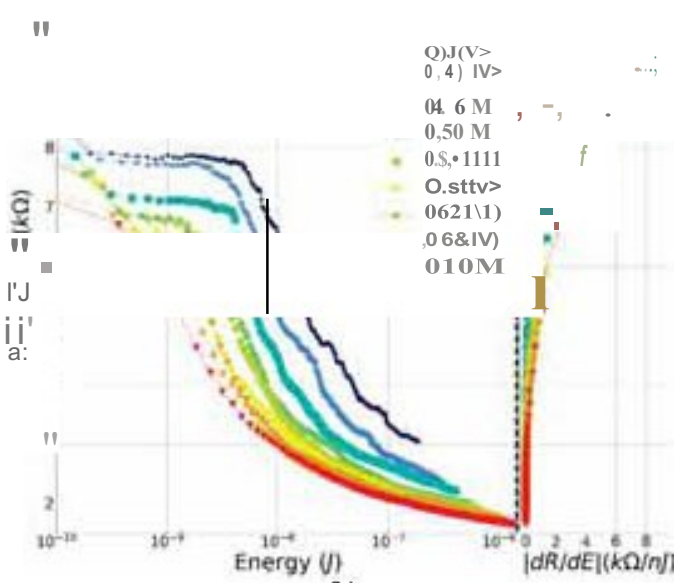

(b)

Fig. 5 Resistmoe evolution; ;naly:;is: (a.) wx.t.tirn.e (p.ilse dura.tim a.:ni (b) w.r.t. theil'gecb?d ewagy.Tu right side ofbothsub-figures sh:,ws th? devioi? clw rate w.r.t. tine (dRAil) and w.x.t.tl-e enagy $(d R / d £)$, CClni'llted for wey resis tan:e valuedulingth?s witi:hingprocess.

This is because, for slowly increasing ramps, especially during the RESET process, there is a significant amount of energy accumulated without affecting the device (considering also the factthat the absolute value of the voltago threshold for RESET is higher than for SET for a particular VRS value, as seen in Fig 3, so it takes longer to cross the threshold voltage and initiate the switching), which is why the results clivergo from the expected in the left side of the plot. Interestingly, these results for the devices of Knowm Inc. are very much in line with other results in (10] and (11] where a totally different device family was considered. Hence, the energy appears to be indeed a universal parameter to be considered for such perfonn anceanalysis.

We conclude this sb.sdy with the results in Fig. 5(a,b) that aim to demonstrate how the switching behavior of the devices can be comprehensively described when energy is the common denominator instead of time. This figure shows the same data shown in Fig. 2 but under a resistance rate change perspective; i.e. the resistance evolution w.r.l (a) time and(b) energy, during the SET process, for a wide set of different amplitudes of the applied square pulse. On the right side, the change rate of the resistance $d R / d t$ (and $d R / d £$ respective! J/, 1 w.r.t. the actual resistance value is shown $\mathrm{As}$ expecteC $\backslash$ we 
notice that the change rate tends to increase with the increasing amplitude of the applied pulses, but still there is a significant dispersion in the obtained data for a particular resistance value. On the other hand, when the same analysis is done w.r.t. the measured energy, the results are very much unified, showing that for a particular resistance value the average change rate per energy unit $d R / d E$ is constant, regardless of the characteristics of the applied pulse. This takes us to the simple conclusion that the energy qualifies as a magnitude capable of characterizing a specific memristive device family as a universal performance parameter.

\section{CONCLUSIONS}

We explored experimentally the SET-RESET behavior of Knowm Inc. bipolar memristors from the energy point of view. Our performance exploration revealed the existence of a switching threshold, to be considered for pulse amplitude selection. However, the energy was proven further a much more comprehensive magnitude, qualifying not only for a better behavioral analysis but also as a universal performance parameter able to characterize different memristive families. From a different point of view, such analysis could give answers to questions such as: "how much energy RS events cost?" and "what is the quantitative effect of the energy on resistance"?

\section{ACKNOWLEDGEMENT}

The authors thank Knowm Inc. for providing the memristor devices used in our experiments.

\section{REFERENCES}

[1] D. B. Strukov, G. S. Snider, D. R. Stewart, and R. S. Williams, "The missing memristor found," Nature, vol. 453, pp. 80 -83, 2008
[2] L. 0. Chua, "Memristor - The Missing Circuit Element," IEEE Trans. Circuit Theory, vol. 18, no. 5, pp. 507-519, 1971

[3] H.-S. P. Wong, et al.," Metal-Oxide RRAM," IEEE Proc., vol. 100, no. 6, pp. 1951-1970,2012

[4] I. Vourkas and G. Ch. Sirakoulis, "Emerging Memristor-based Logic Circuit Design Approaches: A Review," IEEE Circ. and Syst. Mag, vol. 16 , no. 3 (3rd quarter), pp. 15-30, 2016

[5] H. Li T. F. Wu, S. Mitra and H.-S. P. Wong, "Resistive RAM-centric computing: Design and modeling methodology," IEEE Trans. Circuits Syst. I, Reg. Papers, vol. 64, no. 9, pp. 2263-2273, Sep. 2017

[6] I. Vourkas, A. Batsos, and G. Ch. Sirakoulis, "SPICE modeling of nonlinear memristive behavior," Int. J. Circ. Theor. Appl., vol. 43, no. 5, pp. $553-565,2015$

[7] C. Yakopcic, T. M. Taha, G. Subramanyam, and R. E. Pino, "Memristor SPICE Modeling," in Advances in Neuromorphic Memristor Science and Applciations, 1st Ed. The Netherlands: Springer, 2012, pp. 211-244

[8] Y. Pershin and M. Di Ventra "SPICE Model of Memristive Devices with Thresho,ld" Radioengineering, vol. 22, no. 2,pp. 485-489, 2013

[9] Z. Jiang, Y. Wu, S. Yu, L. Yang, K. Song, Z. Karim, and H.-S. Wong, "A Compact Model for Metal-Oxide Resistive Random Access Memory With Experiment Verification," IEEE Trans. Electron Devices, vol. 63, no.5,pp. 1884-18922016

[10] M. Maestro, et al., "Analysis of Set and Reset Mechanisms in NiffifD2based RRAM with Fast Ramped Voltages," Microelectronic Eng., vol. 147, pp. 176-179, 2015

[11] J. Gomez, et al., "Universal Performance Parameters for Resistive Switching Devices," Late Break News session, 2017 IEEE Int. Symp. Circ. Syst.(/SCAS), Baltimore, MD, USA, May 28-31

[12] Knowm Inc., " Ne uromemristive Artificial Intelligence," [Online]. Available: https://knowm.org[Accessed February 2018].

[13] J. Gomez, I. Vourkas, A. Abusleme, and G. Ch. Sirakoulis, "Experimental Measurements on Resistive Switching Devices: Gaining Hands-on Experience," accepted in 2018 Int. Conf on Modern Circuits and Syst. Technol. (MOCAST), Thessaloniki, Greece, May 07-09

[14] F. Alibart, L. Gao, B. D. Hoskins, and D. B. Strukov, " High precision tuning of state for memristive devices by adaptable variation-tolerant algorithm," Nanotechnology, vol. 23 (075201), 2012 